Note: this is a post-print draft of the journal article:

Gillard, F., Dickinson, A., Schneider, U., Taylor, A., Browne, M. (2013) "Multi-pelvis characterisation of articular cartilage geometry". Proceedings of the Institution of Mechanical Engineers Part H: Journal of Engineering in Medicine, 227(12) p1255-1264, DOI: 10.1177/0954411913500265

The final, fully proofed and peer-reviewed journal article is available from the publisher online, via the following link:

http://pih.sagepub.com/content/227/12/1255.full.pdf 


\title{
Multi-pelvis characterisation of articular cartilage geometry
}

\author{
F.C.Gillard ${ }^{1}$, A.S. Dickinson ${ }^{1,3}$, U. Schneider ${ }^{2}$, A.C. Taylor ${ }^{3}$, M. Browne $^{1}$ \\ ${ }^{1}$ Bioengineering Science Research Group, University of Southampton, Highfield, Southampton, \\ Hants, S017 1BJ, UK \\ ${ }^{2}$ Department of Biomechatronic Systems, Fraunhofer Institute IPA Stuttgart, Germany, \\ ${ }^{3}$ Aurora Medical Ltd, Southampton Science Park, Chilworth, Southampton, Hants, S016 7NS, UK
}

\begin{abstract}
The shape of the acetabular cartilage follows the contact stress distribution across the joint. Accurate characterisation of this geometry may be useful for the development of acetabular cup devices that are more biomechanically compliant. In the present study, the geometry of the acetabular cartilage was characterised by taking plaster moulds of the acetabulum from 24 dry bone human pelvises and digitising the mould shapes using a 3D laser scanner. The articular bone surface geometry was analysed, and the shape of the acetabulum was approximated by fitting a best-fit sphere. To test the hypothesis that the acetabulum is non-spherical, a best-fit ellipsoid was also fitted to the geometry. In each case, points around the acetabular notch edge that disclosed the articular surface geometry were identified and vectors were drawn between these and the best-fit sphere or ellipsoid centre. The significantly larger $\mathrm{z}$-radii (into the pole) of the ellipsoids indicated that the acetabulum was non-spherical and could imply that the kinematics of the hip joint is more complex than purely rotational motion and the traditional ball-and-socket replacement may need to be updated to reflect this motion. The acetabular notch edges were observed to be curved, with males exhibiting deeper, wider and shorter notches than females, although the difference was not statistically significant (mean: $p=0.30$ ) and supports the use of non-gender specific models in anatomical studies.
\end{abstract}




\section{INTRODUCTION}

The hip joint is a semi-congruous joint consisting of an acetabular socket which covers the femoral head. The articulating surfaces are covered with hyaline cartilage which plays an important role in lubrication and low friction articulation of the joint $(1,2)$. The cartilage covers the entire surface of the femoral head except for a small region which acts as the attachment site for the ligamentum teres (3). The acetabulum has less coverage and the cartilage exhibits a horseshoe shape which extends around the posterolateral rim leaving the acetabular fossa uncovered $(4,5)$.

The hip joint is exposed to loads ranging from 2.5 times body weight during walking up to eight times body weight on occasion when stumbling (6). Over time, this environment can lead to degeneration of the cartilage underneath both which can have a severe effect on quality of life. Joint degeneration can be treated in a number of ways depending on the condition of the underlying subchondral bone. Traditional treatments for more advanced degeneration include resurfacing and total hip arthroplasty.

Although the cartilage on the acetabular articular surface is resected during total hip arthroplasty, knowledge of its shape and how it varies from patient to patient would be useful in the development of more biomechanically compliant acetabular cup components. The cartilage and articular surface outline is particularly important as mechanical loading influencing cartilage formation $(7,8)$ with the shape of the cartilage being shown to indicate the natural contact stresses across the joint. The acetabulum articular surface is horseshoe shaped, surrounding the central non-articular depression called the acetabular fossa or notch. The horseshoe region is the weight bearing section and it is suggested that its specific shape optimises the contact stress distribution in the hip joint, decreasing the peak contact stress and rendering the stress distribution more uniform $(4,9)$. Total hip arthroplasty components aim to restore the natural biomechanics and loading of the natural joint, however, the insertion of current hemispherical acetabular components have been shown to result in pole contact which is not present in the natural hip even under high loads $(10,11)$. Therefore, novel acetabular components (12-15) that mimic the horseshoe weight bearing shape i.e. the natural geometry are being explored as a potential method for restoring the natural load transfer in the hip after a joint replacement.

The 3D shape of the acetabulum has been a subject of investigation for some time and investigators have used a range of methods including laser scanning $(16,17)$, plaster casting $(18,19)$ and the use of MRI or CT images to 
generate surface models $(20,21)$. From these studies and several others (22-24) it has generally been agreed that the bony articular surface of the acetabulum more closely fits an ellipsoid than a sphere. Nevertheless, the horseshoe outline has yet to be quantified and how it is related to the sphere/ellipsoid fitting technique has not been investigated.

In the present study, a repeatable measurement method for fitting a sphere and ellipsoid to the acetabulum bony articular surface morphology of dry skeletons is implemented in order to identify the best-fitting shape. The study aims to build upon previous work by using the best-fitting procedure to identify and measure the articular surface of the acetabulum using a sphere and an ellipsoid. The study will add information to the literature on the best-fitting shape and aim to provide original data on the outline and quantification of the acetabular notch geometry could be used to inform developers on the horseshoe shape required for biomechanically compliant large diameter acetabular cups for total, hemi- and resurfacing arthroplasty in order to optimise the contact stress distribution in the replaced hip joint. 


\section{METHODS}

24 left acetabulae were analysed, from anonymised human dry bone full- and hemi-pelvis specimens donated with prior informed consent, at Gubener Plastinate $\mathrm{GmbH}$, Guben, Germany. The donor gender of 20 full pelvises was estimated by examining the pelvic girdle (25); 11 were female, 9 male. The remaining 4 hemipelvis donor genders were unknown. The source of the bones and the age at death were unknown. None of the acetabulue were fractured although 5 showed signs of degenerative joint disease.

All morphologic acetabular measurements were performed by one observer (FG) on plaster moulds taken from the left acetabulum on all specimens. Digitised anatomical geometry of each acetabulum was acquired by a 3D laser scanner (Xyris 2000, Taicaan, UK). This system uses a laser triangulation probe with a $30 \mu \mathrm{m}$ laser spot that is scanned across the mould surface to build up a 3D computer image. A 3D point cloud representing each surface was obtained and converted into a .stl standard format in Solidworks 2010 (Dassault Systèmes Solidworks Corps, France) in order to be output as a processed point cloud (.xyz) (Figure 1). To coincide with current methods $(17,26)$ of acetabular morphology assessment a MATLAB program (17) containing a nonlinear least-square algorithm to fit a sphere to the nodal co-ordinate data of the acetabular bearing surface, giving the best fitting acetabulum sphere diameter and centre coordinates. The code was adapted to apply a difference threshold of $+/-0.5 \mathrm{~mm}$ was implemented which deleted points outside this range, eliminating data points from the acetabular notch (below the level of the articular surface) and any error points due to air bubbles that were formed during moulding (Figure 1c). The $0.5 \mathrm{~mm}$ deviation threshold was selected based on previously reported literature data (27) which suggested that there was a $0.5 \mathrm{~mm}$ maximum deviation from spherical of the articular surface. A 3D scatter graph was produced which illustrated the shape of the articular geometry (Figure 1d)

To test the hypothesis that the acetabulum is better described by an ellipsoid than a sphere, the MATLAB program was extended to fit an ellipsoid to the point cloud of each specimen. An ellipsoid was fit to the points cloud using the function ellipsoid_fit.m by Yury Petrov MATLAB Central File Exchange, June $2012^{1}$. The algorithm fits the point cloud to a polynomial expression $\left(\mathrm{Ax}^{2}+\mathrm{By}^{2}+\mathrm{Cz}^{2}+2 \mathrm{Dxy}+2 \mathrm{Exz}+2 \mathrm{Fyz}+2 \mathrm{Gx}+2 \mathrm{Hy}\right.$ $+2 \mathrm{Iz}=1)$ using a linear least square approach and this is solved to find the centre and radii of the ellipse. The horseshoe shape determined in the sphere fitting procedure was used to mask the original point clouds 
(including points in the acetabular notch) and delete points located $3 \mathrm{~mm}$ inside the acetabular notch outline, enabling an ellipsoid to be successfully fitted to the points on the articular surface.

a)

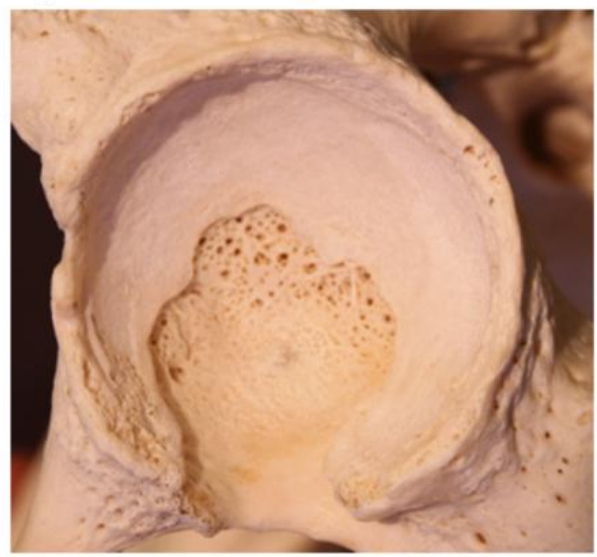

d)

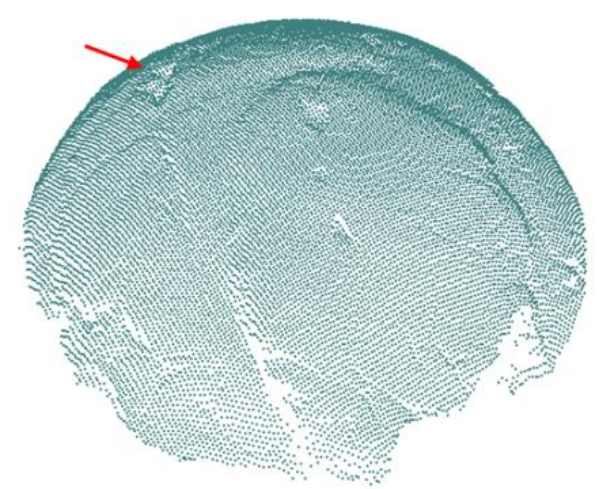

b)

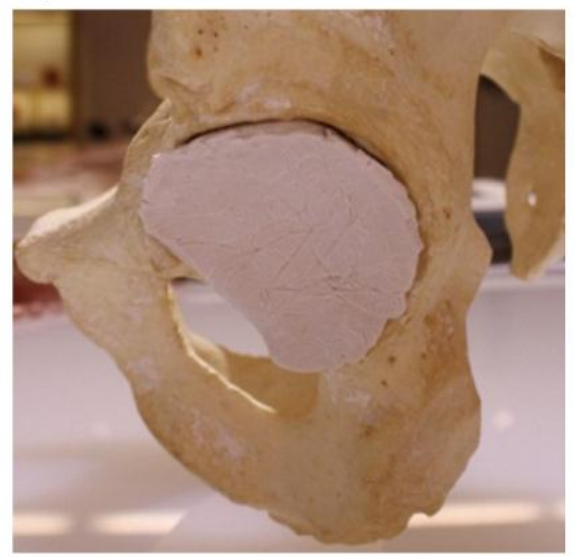

e)

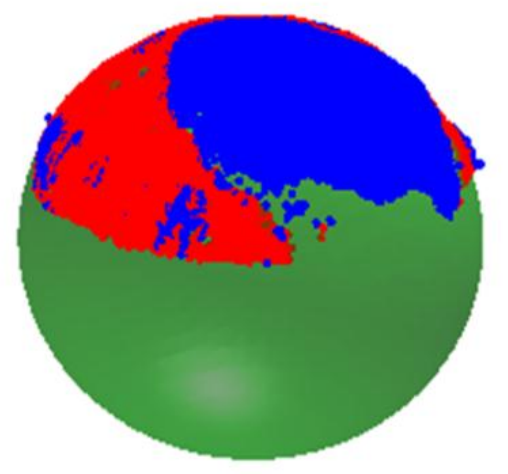

c)

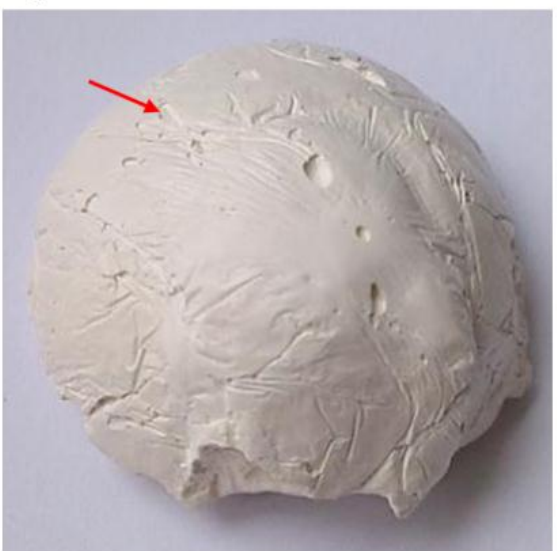

f)

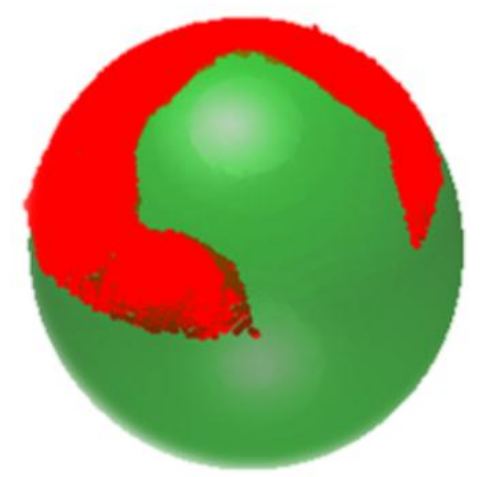

Figure 1: Moulding process of Skeleton Acetabulum (a) involving insertion of plaster (b) to form mould. An air bubble is highlighted by the red arrow (c), generation of surface point cloud of mould using laser scanner (d),ellipsoid/sphere fitting to point cloud (e) and deletion of points outside of error threshold (f).

a)

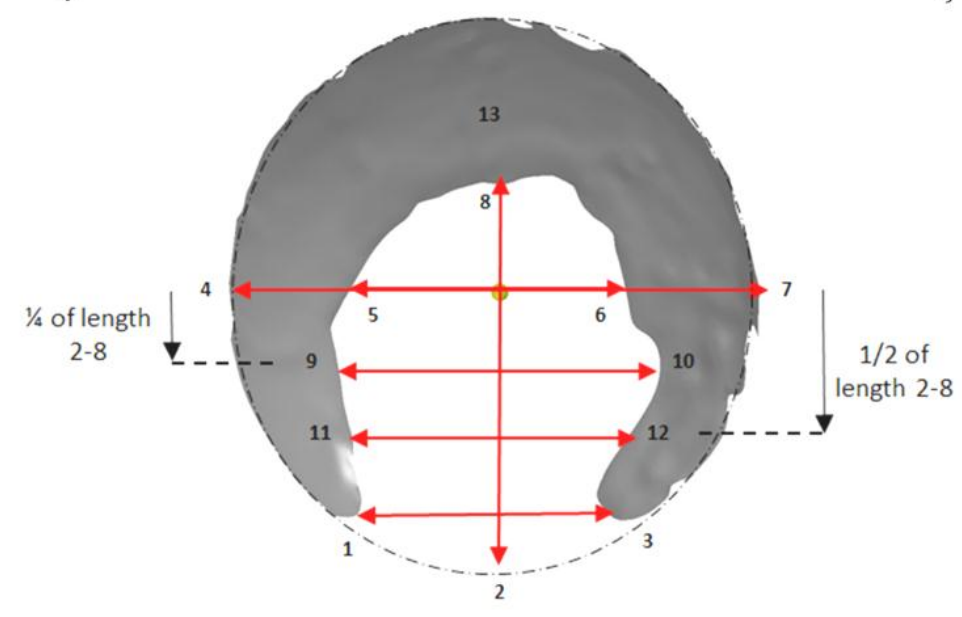

b)

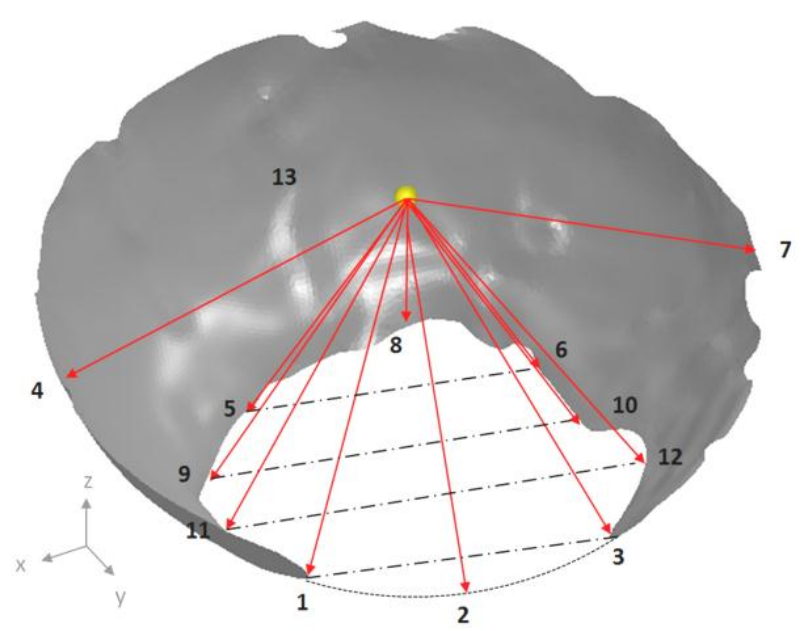

Figure 2: a) Location of points on acetabulum to generate cartilage geometry and b) Angles between location points. 
The ellipsoid axes were aligned along the principle axes of the acetabulae coordinate system (Figure 2) and the radius along each axis was calculated and the ellipsoid centre located. These values were used to calculate the error between the best-fit ellipsoid and each point in the point cloud using the standard ellipsoid equation. To eliminate points within the notch a $+/-0.2 \%(\sim+/-0.5 \mathrm{~mm})$ error threshold was implemented which deleted points outside of this range and exposed the shape of the articular geometry (Figure 1e).

The closeness of the fit of the best-fit sphere and ellipsoid was assessed by calculating the mean deviation between the best-fit sphere/ellipsoid surface and all the points within the point cloud for each acetabulum.

Nine 'landmark' points were picked around the acetabular notch and two from the bony edge of each point cloud produced using the sphere and ellipsoid fitting techniques which eliminate data from the original points that fell within the acetabular notch. All points referenced the best-fit sphere or ellipsoid centre (Table 1,

Figure 2).

Table 1: Dimensions and Angles analysed.

\begin{tabular}{|l|l|}
\hline Acetabulum Points: & Landmarks: \\
\hline $1-3$ & Notch opening length \\
\hline $2-8$ & Notch height through centre \\
\hline $4-7$ & Cartilage width through centre \\
\hline $5-6$ & Notch width through centre \\
\hline $9-10$ & Notch width at a distance equal to quarter of the notch height along y-axis \\
\hline $11-12$ & Notch width at a distance equal to half of the notch height along $y$-axis \\
\hline
\end{tabular}

In three acetabulue the notch did not extend past the centre so points 5 and 6 could not be picked. Vectors between the points and the best-fit sphere or ellipsoid centre were calculated. The distance and angle between corresponding pairs of points (as defined in Table 1), on the outline of the acetabular notch exposed by the bestfit sphere and ellipsoid were then calculated.

The notch opening width, notch height, notch width through the centre and the notch width at set distances from the centre were characterised for each acetabulum and fitting technique. The angle and distance between corresponding points were calculated to give the total angle between points through the sphere or ellipsoid centre.

Reproducibility of the measurement technique was assessed by 5 repeat mouldings on a single acetabulum. 
For comparison against published literature (17), differences between male and female groups were assessed using a two-tailed Student t-test assuming unequal variances. The left acetabulae from the hemi-pelvises were excluded from this section of the study as the gender could not be evaluated using the pelvic girdle technique. 


\section{RESULTS}

The repeatability study of the casting and scanning process showed that the best-fit acetabulum sphere diameter approximation exhibited a range of $+/-0.1 \mathrm{~mm}$ whilst on average the range of the length and angle measurement was $+/-0.5 \mathrm{~mm}$ and $2.1^{\circ}$ respectively when the process was repeated on the same acetabulae. The best-fit ellipsoid approximation exhibited slightly higher deviation with the $\mathrm{x}, \mathrm{y}$ and $\mathrm{z}$ radius exhibiting a range of $+/-0.4,0.4$ and $1.2 \mathrm{~mm}$ whilst on average the range of length and angle measurement was $+/-0.7 \mathrm{~mm}$ and $3.5^{\circ}$ respectively.

Table 2 shows the mean diameter of the fitted spheres and ellipsoids and compares the overall mean male and female diameters. The mean diameter of the spheres fitted to the acetabulae was $50.7 \mathrm{~mm}$ (standard deviation (S.D) of $2.5 \mathrm{~mm}$, range $45.1 \mathrm{~mm}$ to $55.9 \mathrm{~mm}$ ). Males exhibited a larger acetabulum than females averaging 51.6 mm (SD: $2.1 \mathrm{~mm}$ ) compared to $49.6 \mathrm{~mm}$ (SD: $2.5 \mathrm{~mm}$ ). This difference was not significant (p>0.05) (Table 2). The mean diameter along the $\mathrm{x} y$ and $\mathrm{z}$ axes of the fitted ellipsoid were: $\mathrm{x}$ axis $56.4 \mathrm{~mm}$ (standard deviation (S.D) of $6.2 \mathrm{~mm}$, range $47.3 \mathrm{~mm}$ to $74.7 \mathrm{~mm}$ ); y axis $57.2 \mathrm{~mm}$ (standard deviation (S.D) of $6.8 \mathrm{~mm}$, range 47.7 $\mathrm{mm}$ to $77.1 \mathrm{~mm}$ ) and for the $\mathrm{z}$ axis the average radius was $70.0 \mathrm{~mm}$ (standard deviation (S.D) of $16.8 \mathrm{~mm}$, range $47.7 \mathrm{~mm}$ to $90.2 \mathrm{~mm})$. The difference between the $\mathrm{x}$ and $\mathrm{z}$ and $\mathrm{y}$ and $\mathrm{z}$ axes were significant $(\mathrm{p}<0.05)$

(Table 2).

Table 2: Mean diameter of best-fit sphere and ellipsoid and comparison between male and female.

\begin{tabular}{|c|c|c|c|}
\hline & Axes & $\begin{array}{l}\text { Mean best-fit sphere } \\
\text { diameter }(\mathrm{mm})\end{array}$ & $\begin{array}{c}\text { Mean best-fit ellipsoid } \\
\text { diameter }(\mathrm{mm})\end{array}$ \\
\hline \multirow{3}{*}{ Mean } & $x$ & \multirow{3}{*}{$50.7 \pm 2.5$} & $56.4 \pm 6.2$ \\
\hline & $y$ & & $57.2 \pm 6.8$ \\
\hline & $z$ & & $70.0 \pm 16.8$ \\
\hline \multirow{3}{*}{ Males } & $x$ & \multirow{3}{*}{$51.6 \pm 2.1$} & $56.2 \pm 3.0$ \\
\hline & $y$ & & $57.0 \pm 3.0$ \\
\hline & z & & $70.0 \pm 8.2$ \\
\hline \multirow{3}{*}{ Females } & $x$ & \multirow{3}{*}{$49.6 \pm 2.5$} & $56.4 \pm 8.0$ \\
\hline & $y$ & & $57.2 \pm 8.8$ \\
\hline & z & & $68.2 \pm 18.4$ \\
\hline
\end{tabular}


Male and female $\mathrm{x}$ and y ellipsoid diameters were similar averaging $56.2 \mathrm{~mm}$ (SD: $3.0 \mathrm{~mm}$ ) compared to 56.4 $\mathrm{mm}(\mathrm{SD}: 8.0 \mathrm{~mm}$ ) in the $\mathrm{x}$ axis and $57.0 \mathrm{~mm}(\mathrm{SD}: 3.0 \mathrm{~mm}$ ) compared to $57.2 \mathrm{~mm}$ (SD: $8.8 \mathrm{~mm})$ in the $\mathrm{y}$ axis. There was a greater difference in the $\mathrm{z}$ axis in which the males average was $70.0 \mathrm{~mm}$ (SD: $8.2 \mathrm{~mm}$ ) compared to the female average which was $68.2 \mathrm{~mm}$ (SD: $18.4 \mathrm{~mm}$ ). These gender differences were not significant $(\mathrm{p}>0.05)$.

The mean deviation of all surface points from the best-fit sphere and ellipsoid surface was $1.3 \mathrm{~mm}$ (SD: 0.3 $\mathrm{mm})$ and $0.4 \mathrm{~mm}(\mathrm{SD}: 0.2 \mathrm{~mm})$ respectively. This decrease in deviation was significantly different.

The results for the lengths and angles between the selected points for both the sphere and ellipsoid analyses are shown in Table 3. In both cases the only angle larger than $90^{\circ}$ was the cartilage width angle. Three acetabulae were excluded from each study as the notch width and angle through the central axes as the notch did not extend past the sphere centre; these were all females.

Table 3: Mean Calculated Values for Acetabulum Points fitted with Best-fit Sphere and Ellipsoid

\begin{tabular}{|c|c|c|c|c|}
\hline & \multicolumn{2}{|c|}{ Best-fit Sphere } & \multicolumn{2}{c|}{ Best-fit Ellipsoid } \\
\hline $\begin{array}{c}\text { Acetabulum } \\
\text { Points }\end{array}$ & $\begin{array}{c}\text { Length between } \\
\text { points (mm) }\end{array}$ & Angle $\left(^{\circ}\right)$ & $\begin{array}{c}\text { Length between } \\
\text { points }(\mathrm{mm})\end{array}$ & Angle $\left(^{\circ}\right)$ \\
\hline $1-3$ & $23.0 \pm 3.1$ & $53.5 \pm 7.0$ & $23.8 \pm 5.7$ & $68.3 \pm 26.6$ \\
\hline $2-8$ & $32.8 \pm 2.1$ & $75.6 \pm 6.2$ & $29.3 \pm 3.0$ & $78.4 \pm 9.4$ \\
\hline $4-7$ & $44.7 \pm 1.8$ & $131.3 \pm 6.4$ & $44.3 \pm 9.4$ & $97.6 \pm 31.6$ \\
\hline $5-6$ & $22.7 \pm 3.6$ & $52.6 \pm 8.8$ & $24.5 \pm 3.9$ & $45.2 \pm 11.1$ \\
\hline $9-10$ & $28.7 \pm 2.6$ & $68.3 \pm 7.1$ & $28.7 \pm 6.4$ & $54.8 \pm 17.4$ \\
\hline $11-12$ & $27.2 \pm 3.7$ & $64.6 \pm 7.7$ & $27.8 \pm 6.3$ & $58.9 \pm 20.1$ \\
\hline
\end{tabular}

Both analyses demonstrated a difference between the mean notch opening length and the notch width through the centre but this was not significantly different (Sphere $p=0.40$, Ellipsoid $p=0.84$ ). In both cases the mean notch width and angle at a distance away from the centre equal to a quarter and half of the overall notch height were both significantly larger than the mean notch width through the centre $(\mathrm{p}<0.05)$ and the notch opening length $(\mathrm{p}<0.05)$ (Table 3). 


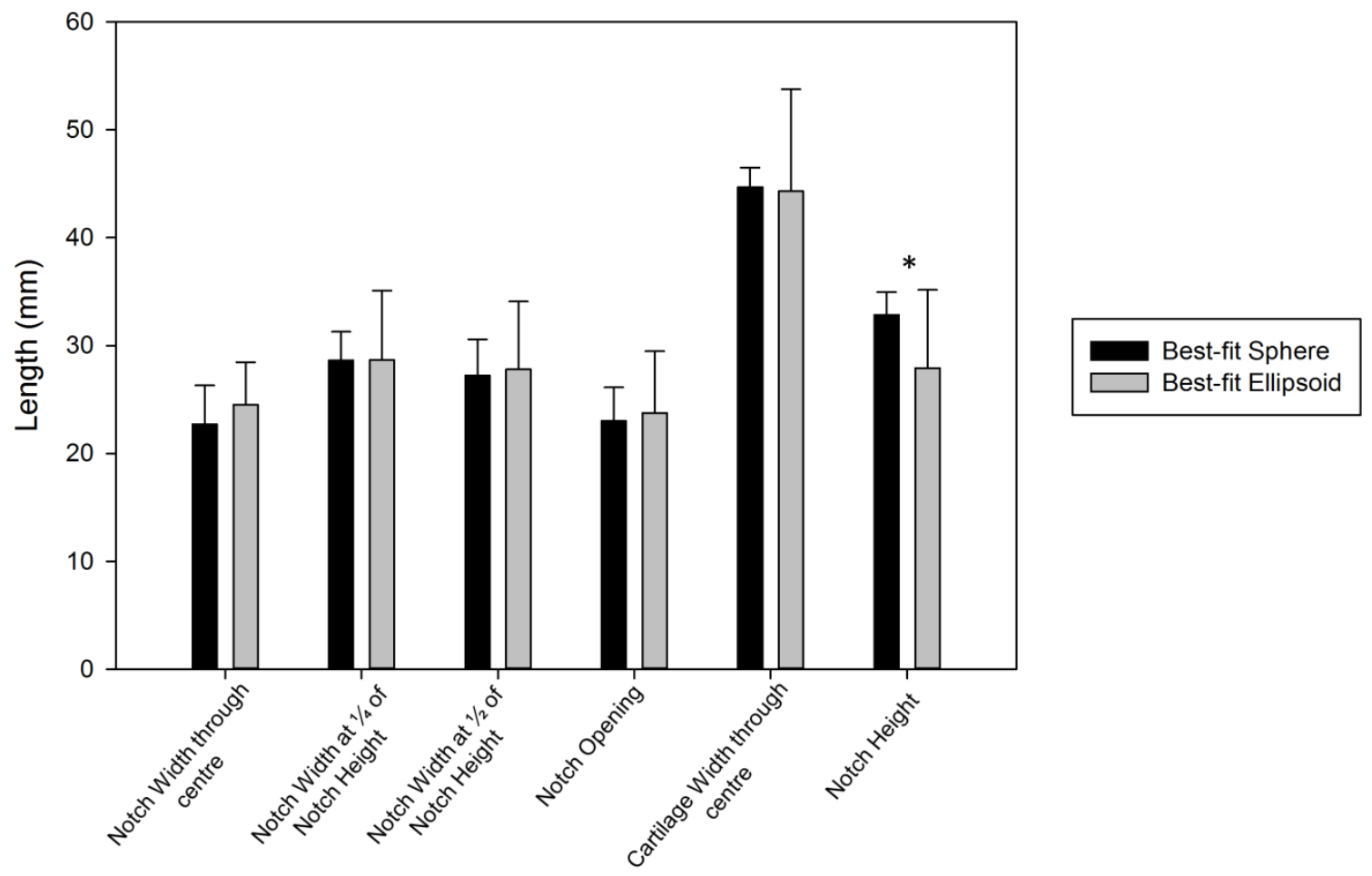

Figure 3: Mean Acetabulum dimensions between six points for best-fit sphere and ellipsoid, (* indicates significant difference between values)

The width geometry calculated for the best-fit sphere were calculated on average to be smaller than the best-fit ellipsoid (Figure 3), although these differences were not significant ( $p>0.05)$. The calculated angles and notch heights for the best-fit sphere and ellipsoid were all significantly different (Figure 3 and Figure 4).

Males exhibited larger width dimensions than the female group, for both the sphere and ellipsoid analyses (Figure 5, Figure 6). For the best-fit sphere analysis these were all significantly difference except the cartilage width and notch height (Figure 5a, Figure 6a). However, only the notch width at half the notch height was significantly different for the best-fit ellipsoid (Figure 5b, Figure $\mathbf{6 b}$ ). Females exhibit longer notches than males for both analyses however the difference was not significant. 


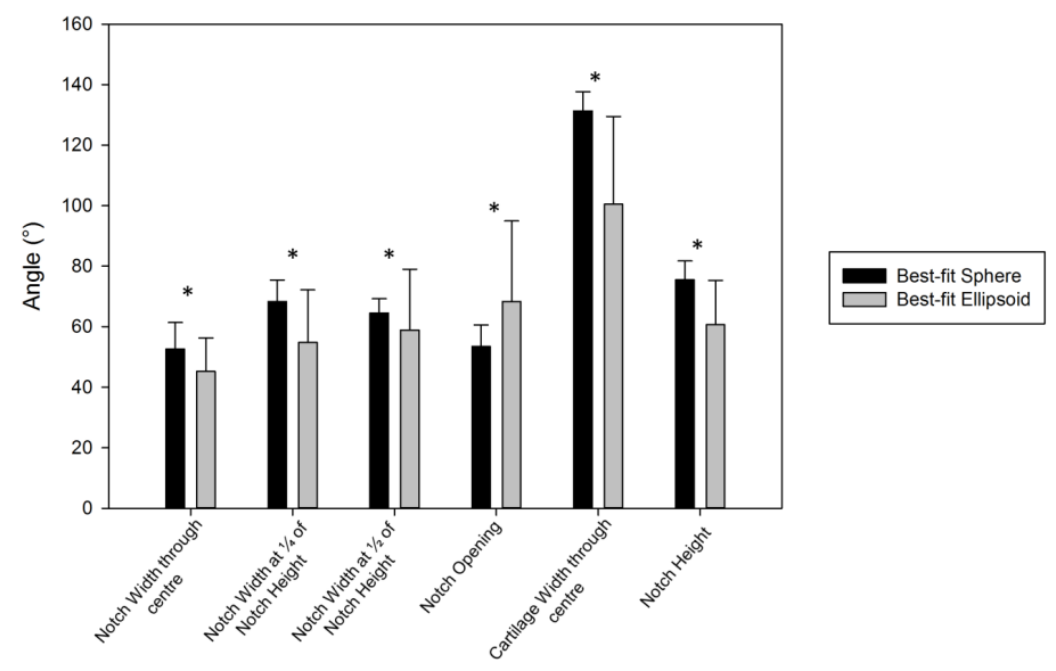

Figure 4: Mean Acetabulum Angles between six points for best-fit sphere and ellipsoid, (* indicates significant difference between values)
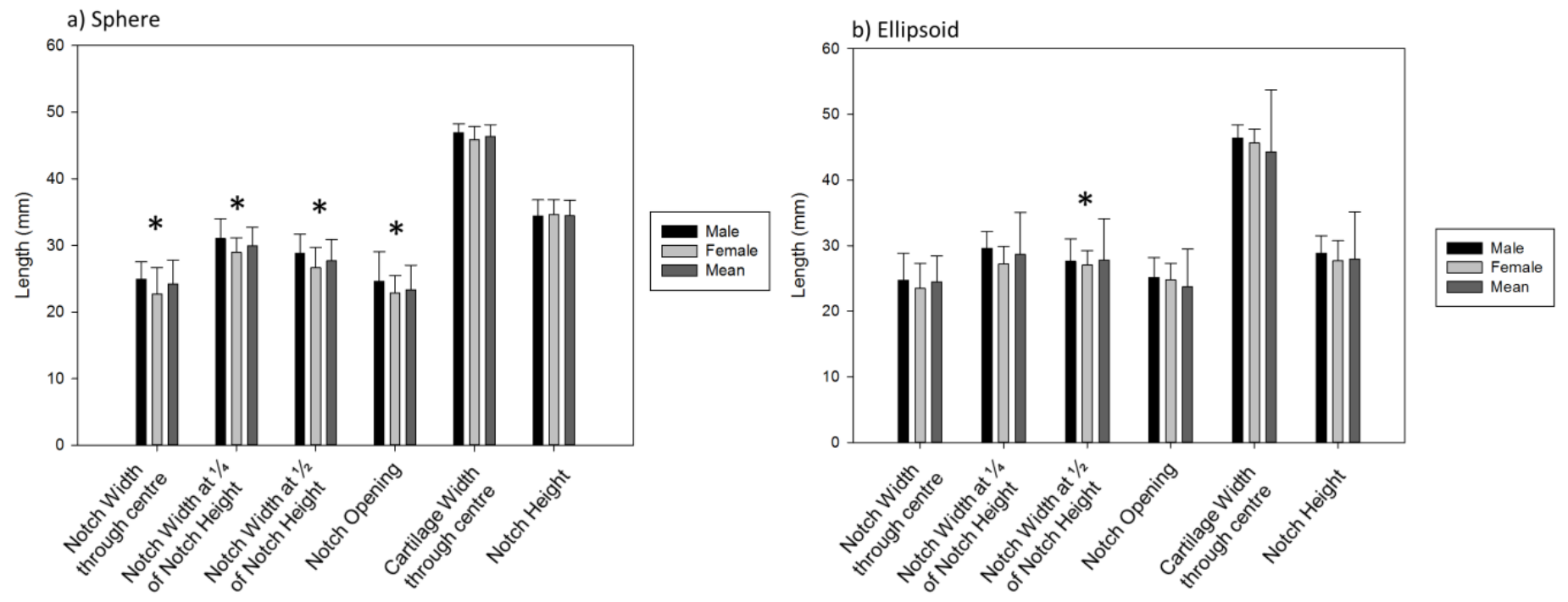

Figure 5: Width between chosen points in acetabulum of best-fit sphere (a) and best-fit ellipsoid (b). Overall mean and mean male and female are compared.

(* indicates significant difference between values) 

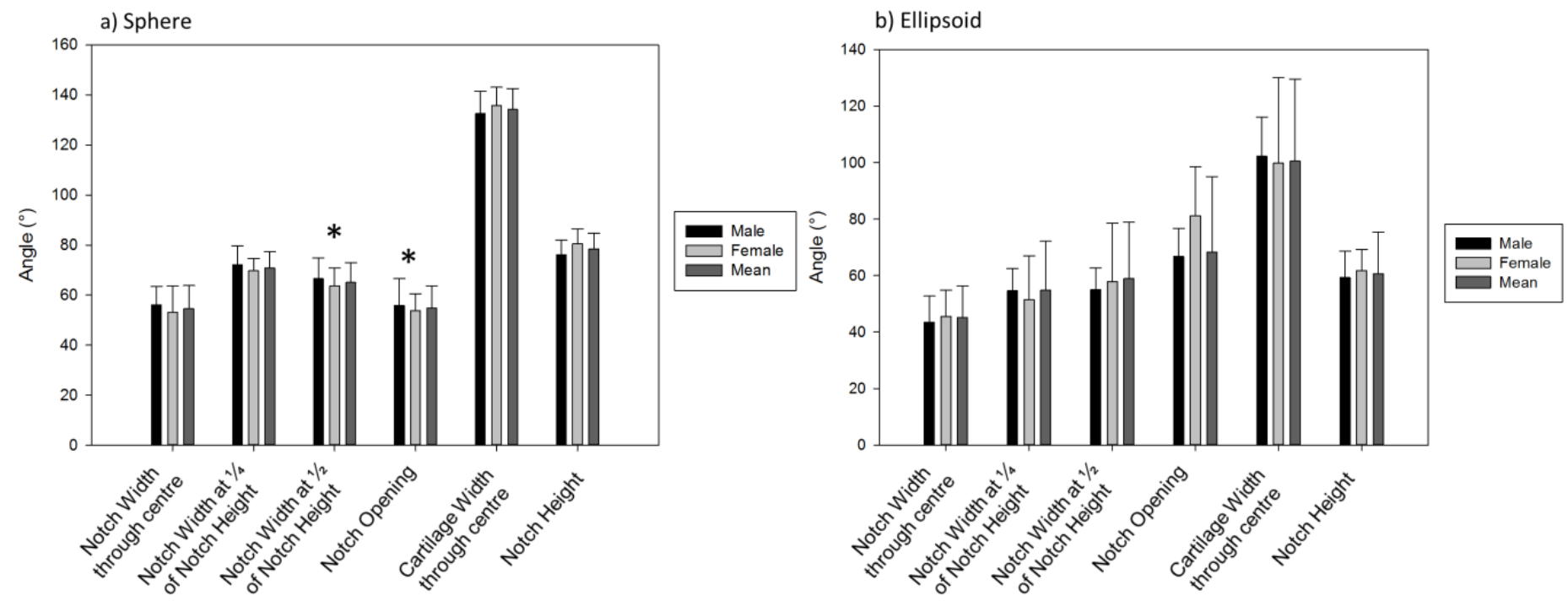

Figure 6: Angles between chosen points in acetabulum of best-fit sphere (a) and best-fit ellipsoid (b). Overall mean and mean male and female are compared.

(* indicates significant difference between values)

\section{DISCUSSION}

This study aimed to assess the variability of the acetabulum articular surface geometry using a sample size of 24 left acetabulae. Based on existing techniques (16-19), a best-fit sphere was fitted to the acetabulum which highlighted how the acetabular geometry varies with gender. To confirm whether the acetabulum articular surface more closely fits an ellipsoid, a similar analysis was employed using an ellipsoid fitted to the acetabulum articular surface.

Both techniques allowed the horseshoe shape of the acetabulum to be exposed, but it was clear that the ellipse fitted more closely to the data: firstly the ellipsoid had more points located closer to its surface than the sphere. The mean deviation between the fitted ellipsoid and the acetabulum was $31 \%$ of the mean deviation between the fitted sphere and the acetabulum. Secondly, the ellipsoid radii along the $\mathrm{x}$ and $\mathrm{y}$ principle axes were similar with an average difference of $0.6 \mathrm{~mm}$. However, the radius along the z-axis was significantly larger than the other axes which confirmed the suggested non-spherical nature of the acetabulum. The relationship obtained between the ellipsoid radii proposes that the acetabulum roughly fits a prolate spheroid as the polar axis (z-axis) is greater than the equatorial diameter ( $\mathrm{x} \& \mathrm{y}$ axes).

The height, width and corresponding angle measurements revealed new information relating to the acetabular notch morphology and highlighted differences between fitting a sphere and an ellipsoid to the data. For each analysis, regardless of gender, the notch opening width and the width through the centre of the sphere/ellipsoid 
were not significantly different. Instead the width increased to a maximum at a distance approximately a quarter of the notch height below the best-fit shape centre. This equated to a subtended angle of $16.7^{\circ}+/-1.6^{\circ}$ and $19.7^{\circ}$ $+/-1.5^{\circ}$ in front of the ellipsoid and sphere centre respectively. This is consistent with the study by Oberländer \& Kurrat (28) which concluded that the widest region of the notch was about $15^{\circ}$ degrees away from the acetabular roof.

The significant differences between the geometry obtained from fitting an ellipsoid and a sphere were observed when assessing the notch height and angles between points. The height of the notch was on average larger for the sphere fit which may be attributed to the sphere fitting technique removing more data points from the acetabular notch near the pole of the acetabulum. The angles are generally smaller for the ellipsoid than for the sphere due to the larger z-radius of the ellipsoid. This means the centre of the ellipsoid is located further away (along the $\mathrm{z}$ axes) from the pole of the acetabulum compared to the sphere's centre location. This difference does not significantly affect width calculations (Table 3) due to similarities in $\mathrm{x}$ and $\mathrm{y}$ centre coordinates of the ellipsoid and sphere. However, for accurate angle calculations which reference the centre an ellipsoid should be fitted to the acetabulum morphology.

The implications of these findings are relevant for the design of acetabular components for a number of reasons. Firstly, the ellipsoid nature of the articular surface could imply the kinematics of the hip joint may be more complex than purely rotational motion exhibited by a ball-and-socket joint. Secondly, the determination that the ellipsoid centre is further away from the articular surface than previously suggested by the sphere could have implications on joint replacements. It is critical for the hip centre to be correctly reconstructed after a replacement in order to restore the normal biomechanics of the joint and avoid impingement between the proximal femur or femoral component (29). Based on these findings the traditional ball-and-socket replacement joint may need to be updated to ensure correct joint biomechanics are achieved however this is a suggestion and requires further investigation.

Finally, the determination of the outline of the acetabular notch in this study is useful for the development of novel horseshoe shaped components (12-15) which aim to more effectively load the acetabular structures (30). The MITCH PCR cup (Stryker Orthopedics Corp, Mahwah, USA) explored by Latif et al. (12) is an example; the component is horseshoe shaped and made from a polymer composite. Studies $(12,13)$ have illustrated that the cup loaded the acetabulum more effectively and without pole contact compared to the hemispherical design. 
This was partly due to the material which exhibited a modulus closer to bone, however it was also due to the design restricting the areas of bone through which load was transferred. The horseshoe shape ensured load was transferred onto the cranial region of the acetabulum, the posterior-inferior region at the ischial facet and the anterior region, each an area outside of the acetabular notch. Although the studies assessed load transfer in the acetabulum they did not quantify the horseshoe shape which was the basis of their biomechanically compliant implants. It has been shown that the notch's geometry contributes to a more uniform articular contact stress distribution over the horseshoe section (4) and its size can shift the stress pole and increase the peak contact stress in the joint if not correctly determined.

The influence of gender on acetabulum morphology was also explored in this study. The study showed that males exhibited larger acetabulae than females when fitted with a sphere and ellipsoid. Although this result was not statistically significant, similar sphere fitting studies $(17,26)$ have shown a significant difference between gender. In addition to acetabulum size, both the sphere and ellipsoid analyses observed differences to suggest males exhibit wider, shorter and deeper acetabular notches. All differences were significant for the best-fit sphere except the cartilage width and notch height. However, only the notch width at half the notch height was significantly different for the best-fit ellipsoid. Köhnlein et al. (19) reported wider notches in females, however our data correlates with that of Oberländer \& Kurrat (28) which suggests no gender dependency enabling an average acetabulum to be calculated. The difference in outcomes between this study and similar acetabulum morphology studies $(17,19,26)$ could be attributed to a number of factors. Firstly, this study adopted a computational approach for measurement whereas Köhnlein et al. (19) used manual measurement techniques. Secondly, different centres were used to obtain moulds and no patient ethnicity details could be obtained for the specimens used in this study (although $84 \%$ of the centre's donors are of German nationality (31)). Previous morphological studies have shown that ethnicity affects anthropometric dimensions (32-34). Similar sphere fitting studies have been conducted on Caucasian, Afro-Caribbean (26) and western European donors $(17,19)$ although the latter were obtained from archaeological sites and the actual ethnicity can only be presumed. An extension of this work would be to conduct a repeat of this investigation on acetabulae of known ethnicity to assess this relationship.

Although the gender study established a difference between acetabulum size, no significant difference between male and female acetabulum cartilage geometry was established for the ellipsoid study. This supports the use of 
acetabulum models in anatomical studies that exhibit average notch geometries for a given acetabulum size regardless of gender.

The study and the results must be interpreted with consideration of their strengths and limitations. The plaster moulding technique was adopted in this study as it is considered the gold standard in orthodontic treatment due to its high accuracy and reliability (19). This was reflected by the results of the repeatability analysis performed in this study. In addition the study was carried out by one observer who performed the mould preparation and conducted the computational analysis, eliminating interobserver effects.

A novel aspect of this investigation is in applying both spherical and ellipsoid geometries to the acetabulum surface and using vectors to calculate the angles and corresponding lengths. Each vector referenced the best-fit ellipsoid or sphere centre which compensated for any difference in mould orientation during scanning.

The number and form of the acetabulae used in this study limits the strength of the relationships obtained as data was ignored for the acetabular cup groups that contained one sample e.g. extreme sizes. A larger cohort should avoid this limitation and generate results over a larger size range. With sufficient input samples, statistical shape modelling (SSM) and principal component analysis (PCA) could be applied to the current data set (35-37) for further analysis. The repeatability of the plaster cast method was analysed, concluding a small amount of variation which could be due to the operator. Nevertheless, comparative studies have suggested no differences between computer-guided and manual measures (38). A factor which was not investigated in this study was the effect of the number of digitalised points taken over the surface of the plaster mould. In this study a spacing of $0.33 \mathrm{~mm}$ was selected between points and it is suggested that a smaller spacing between points could improve the accuracy of the results. An extension of this work would be to conduct a repeat of this investigation using varying number of points on the surface of the mould.

In addition, shrinkage of bone during the drying process has been identified in a number of studies on the skull (39), femur (40), radius and ulna (41). It is possible that the dry acetabulae used in this study contracted during the drying process, but there is precedence for the use of dry bones for acetabular analysis $(17,19)$, and if shrinkage is relatively uniform, the relative anatomic trends observed in this study would still hold.

In addition to aiding implant design it has been suggested that characterising the acetabulum cartilage morphology could aid cartilage regeneration techniques (42). Current methods are limited by availability of donor tissue sources, donor site morbidity and prolonged rehabilitation times. Therefore second-generation 
tissue-engineered cartilaginous constructs which match the complex surface geometries of larger articular cartilage defects have been suggested to improve healing (42). Recently the hip has been suggested as a potential site for biologic resurfacing using shaped cartilaginous constructs in which a concave impression would be used to reconstruct the degenerated acetabulum (43). The main complication is that any surface incongruity between these grafts and the surrounding native cartilage surface has been shown to result in local mechanical stresses that may be unfavourable on the success of the graft treatment. Therefore the graft must be anatomically shaped $(42,43)$ and based on quantified geometrical data of the hip joint in order to promote their success. In conjunction with cartilage thickness studies, the methodology outlining the acetabular notch described in this study could be extended to surface models generated from medical images to provide the geometrical data required to produce more anatomically shaped constructs.

In conclusion this study has confirmed that while the sphere fitting technique can be used to calculate distances between points in the acetabulum, the best-fit ellipsoid better characterised the acetabulum geometry. This analysis has established relationships between the acetabulum geometry and supports the use of acetabulum models in anatomical studies that exhibit average notch geometries regardless of gender.

\section{CONFLICT OF INTEREST}

None

\section{ACKNOWLEDGMENTS}

This study was funded by a European Union $7^{\text {th }}$ Framework programme (Project No. 232151 'EnDuRE'). The authors would like to thank the Gubener Plastinate GmbH, Guben, Germany for allowing access to the skeletal specimens.

\section{ETHICAL APPROVAL}

All donor samples used in the study were obtained with prior, written, informed consent by the Institute for Plastination (IfP) in which the donors agreed for any part of their body to be used provided they served medical research or training or 
offer medical treatment by doctors and medical institutions. All donated samples obtained by the IfP were accounted for in the mortality papers held by the Notar in the City of Heidelberg, and each donation was approved with a separate legal case number which certified that the IfP have obtained consent for their use.

${ }^{1}$ Yury Petrov, http://www.mathworks.com/matlabcentral/fileexchange/24693- ellipsoid-fit. 


\section{REFERENCES}

1. Athanasiou K, Darling EM, Hu JC. Articular cartilage tissue engineering - Chapter 1: Morgan \& Claypool Publishers; 2009.

2. Bronzino JD. The biomedical engineering handbook. 1 - Chapter 20: Springer; 2000.

3. Gray H, Holmes T. Anatomy, descriptive and surgical: Longman, Green, Longman, Roberts and Green; 1864.

4. Daniel M, Iglic A, Kralj-Iglic V. The shape of acetabular cartilage optimizes hip contact stress distribution. J Anat. 2005;207(1):85-91.

5. Daniel M, Sochor M, Iglic A, Kralj-Iglic V. Hypothesis of regulation of hip joint cartilage activity by mechanical loading. Med Hypotheses. 2003;60(6):936-7.

6. Bergmann G, Graichen F, Rohlmann A. Hip joint contact forces during stumbling. Langenbeck Arch Surg. 2004;389(1):53-9.

7. Arokoski JPA, Jurvelin JS, Vaatainen U, Helminen HJ. Normal and pathological adaptations of articular cartilage to joint loading. Scand J Med Sci Spor. 2000;10(4):186-98.

8. Guilak F, Ratcliffe A, Mow VC. Chondrocyte deformation and local tissue strain in articular cartilage: A confocal microscopy study. J Orthopaed Res. $1995 ; 13(3): 410-21$

9. Gu DY, Dai KR, Hu F, Chen YZ. The Shape of the Acetabular Cartilage Surface and its Role in Hip Joint Contact Stress. 2010 Annual International Conference of the Ieee Engineering in Medicine and Biology Society (Embc). 2010:3934-7.

10. Widmer KH, Zurfluh B, Morscher EW. Load transfer and fixation mode of press-fit acetabular sockets. J Arthroplasty. 2002;17(7):926-35. Epub 2002/10/11.

11. Manley M, Ong K, Kurtz S, Rushton N, Field R. BIOMECHANICAL ASSESSMENT OF A NOVEL DEFORMABLE ACETABULAR CUP DESIGN. Journal of Bone \& Joint Surgery, British Volume. 2008;90-B(SUPP II):304.

12. Latif AMH, Mehats A, Elcocks M, Rushton N, Field RE, Jones E. Pre-clinical studies to validate the MITCH PCR (TM) Cup: a flexible and anatomically shaped acetabular component with novel bearing characteristics. J Mater Sci-Mater M. 2008;19(4):1729-36.

13. Field RE, D Cronin M, Singh PJ, Burtenshaw C, Rushton N. Bone remodeling around the Cambridge cup - A DEXA study of 50 hips over 2 years. Acta Orthop. 2006;77(5):726-32.

14. Field RE, Rajakulendran K, Eswaramoorthy VK, Rushton N. Three-year prospective clinical and radiological results of a new flexible horseshoe acetabular cup Hip international : the journal of clinical and experimental research on hip pathology and therapy. 2012;22(6):598-606. Epub 2012/12/13.

15. Field RE, Rushton N. Five-year clinical, radiological and postmortem results of the Cambridge Cup in patients with displaced fractures of the neck of the femur. J Bone Joint Surg Br. 2005;87-B(10):1344-51

16. Gu DY, Chen YZ, Dai KR, Zhang S, Yuan JB. The shape of the acetabular cartilage surface: A geometric morphometric study using three-dimensional scanning Med Eng Phys. 2008;30(8):1024-31.

17. Thompson MS, Dawson T, Kuiper JH, Northmore-Ball MD, Tanner KE. Acetabular morphology and resurfacing design. Journal of Biomechanics. 2000;33(12):1645-53.

18. Menschik F. The hip joint as a conchoid shape. Journal of Biomechanics. 1997;30(9):971-3.

19. Kohnlein W, Ganz R, Impellizzeri FM, Leunig M. Acetabular Morphology: Implications for Joint-preserving Surgery. Clin Orthop Relat R. 2009;467(3):682-91.

20. Rasquinha BJ, Sayani J, Rudan JF, Wood GCA, Ellis RE. Articular surface remodeling of the hip after periacetabular osteotomy. Int J Comput Ass Rad. 2012;7(2):241-8.

21. Pienkowski D, Resig J, Talwalkar V, Tylkowski C. Novel Three-Dimensional MRI Technique for Study of Cartilaginous Hip Surfaces in Legg-Calve-Perthes Disease. J Orthopaed Res. 2009;27(8):981-8.

22. Greenwald A, Oconnor JJ. Transmission of Forces through Human Hip-Joint. Phys Med Biol. 1971;16(2):339-\&.

23. Afoke N, Byers P, Hutton W. Contact pressures in the human hip joint. Journal of Bone \& Joint Surgery, British Volume. 1987;69-B(4):536-41.

24. von Eisenhart R, Adam C, Steinlechner M, Müller-Gerbl M, Eckstein F. Quantitative determination of joint incongruity and pressure distribution during simulated gait and cartilage thickness in the human hip joint. J Orthopaed Res. 1999;17(4):532-9.

25. Krogman WM. The human skeleton in forensic medicine: C.C. Thomas; 1962.

26. Murtha PE, Hafez MA, Jaramaz B, DiGioia AM, 3rd. Variations in acetabular anatomy with reference to total hip replacement. J Bone Joint Surg Br. 2008;90(3):308-13. Epub 2008/03/04

27. Rushfeldt PD, Mann RW, Harris WH. Improved techniques for measuring in vitro the geometry and pressure distribution in the human acetabulum--I. Ultrasonic measurement of acetabular surfaces, sphericity and cartilage thickness. J Biomech. 1981;14(4):253-60. Epub 1981/01/01.

28. Oberlander W, Kurrat HJ, Breul R. Examination of Extension of Osseous Facies Lunata - Functional Study. Z Orthop Grenzgeb. 1978;116(5):675-82.

29. Callaghan JJ, Rosenberg AG, Rubash HE. The Adult Hip: Lippincott Williams \& Wilkins; 2007.

30. Manley MT, Ong KL, Kurtz SM. The potential for bone loss in acetabular structures following THA. Clin Orthop Relat R. $2006(453): 246-53$.

31. GmbH GP. Body Donation. 2012 [cited 2012 28th August]; Available from: <http://www.plastinarium.de/en/plastinarium_e/body_donation.html>

32. Nelson DA, Pettifor JM, Barondess DA, Cody DD, Uusi-Rasi K, Beck TJ. Comparison of cross-sectional geometry of the proximal femur in white and black women from Detroit and Johannesburg. J Bone Miner Res. 2004;19(4):560-5.

33. Rawal BR, Ribeiro R, Malhotra R, Bhatnagar N. Anthropometric measurements to design best-fit femoral stem for the Indian population. Indian J Orthop. 2012;46(1):46-53.

34. Theobald TM, Cauley JA, Gluer CC, Bunker CH, Ukoli FAM, Genant HK, et al. Black-white differences in hip geometry. Osteoporosis Int. 1998;8(1):61-7.

35. Bryan R, Mohan PS, Hopkins A, Galloway F, Taylor M, Nair PB. Statistical modelling of the whole human femur incorporating geometric and material properties. Med Eng Phys. 2010;32(1):57-65.

36. Heimann T, Meinzer HP. Statistical shape models for 3D medical image segmentation: A review. Med Image Anal. 2009;13(4):543-63.

37. Rajamani KT, Styner MA, Talib H, Zheng GY, Nolte LP, Ballester MAG. Statistical deformable bone models for robust 3D surface extrapolation from sparse data. Med Image Anal. 2007;11(2):99-109.

38. Stevens DR, Flores-Mir C, Nebbe B, Raboud DW, Heo G, Major PW. Validity, reliability, and reproducibility of plaster vs digital study models: Comparison of peer assessment rating and Bolton analysis and their constituent measurements. Am J Orthod Dentofac. 2006;129(6):794-803.

39. Todd TW. The Effect of Maceration and Drying upon the Linear Dimensions of the Green Skull. J Anat. 1923;57(Pt 4):336-56. Epub 1923/07/01.

40. Trotter M, Gleser GC. Estimation of Stature from Long Limb Bones of American Whites and Negroes - Reply. Am J Phys Anthropol. 1977;47(2):355-6.

41. Athawale MC. Estimation of Height from Lengths of Forearm Bones. A Study of One Hundred Maharashtrian Male Adults of Ages between Twenty-Five and Thirty Years. Am J Phys Anthropol. 1963;21:105-12. Epub 1963/06/01.

42. Han EH, Bae WC, Hsieh-Bonassera ND, Wong VW, Schumacher BL, Gortz S, et al. Shaped, stratified, scaffold-free grafts for articular cartilage defects. Clin Orthop Relat R. 2008;466(8):1912-20.

43. Koh JL, Wirsing K, Lautenschlager E, Zhang LO. The effect of graft height mismatch on contact pressure following osteochondral grafting - A biomechanical study. Am J Sport Med. 2004;32(2):317-20. 